\title{
A Rapid Controller of Temperature for Use in Determining Arrhenius Profiles in Biomembrane Systems
}

\author{
Frederic L. Hoch ${ }^{1}$ and Bo Höijer ${ }^{2}$ \\ Received March 20, 1980; revised August 5, 1980
}

\begin{abstract}
To minimize artifacts in temperature-velocity (Arrhenius) profiles due to aging of preparations of biological membranes, a rapid controller of temperature was developed for spectrophotometric or polarographic $\left(\mathrm{O}_{2}\right.$ electrode) measurements. The reaction mixture is cooled or heated through contact with Peltier elements. One Pt temperature sensor in the cuvette or electrode holder controls current flow into the Peltier units, and another Pt temperature sensor in the reaction mixture is used to read out the sample temperature on a meter or recorder, and to provide feedback control. The sample temperature can be reproducibly set to within $0.1^{\circ} \mathrm{C}$, with a noise level of $0.04^{\circ} \mathrm{C}$ or less; a change of $4^{\circ} \mathrm{C}$ takes 1 min.
\end{abstract}

Key Words: Rapid temperature regulator; Arrhenius plot profiles; biomembranes.

\section{Introduction}

Alterations in the Arrhenius plots of velocity data on membrane-dependent enzyme and transporter systems are informative in indicating changed protein-lipid interactions (Raison, 1973; Fox, 1975). Certain technical problems arise in measuring velocities at different temperatures. Because of the variance between preparations of similar biological membranes, it is preferable to plot sufficient (at least 10) data points obtained from a single preparation. But such preparations age, and change both absolute rates and

\footnotetext{
${ }^{\mathrm{t}}$ Departments of Internal Medicine and Biological Chemistry, The University of Michigan Medical School, Ann Arbor, Michigan 48 109. On leave at the Arrhenius Laboratory, University of Stockholm.

${ }^{2}$ Department of Biochemistry, Arrhenius Laboratory, University of Stockholm, S-106 91, Stockholm, Sweden.
} 


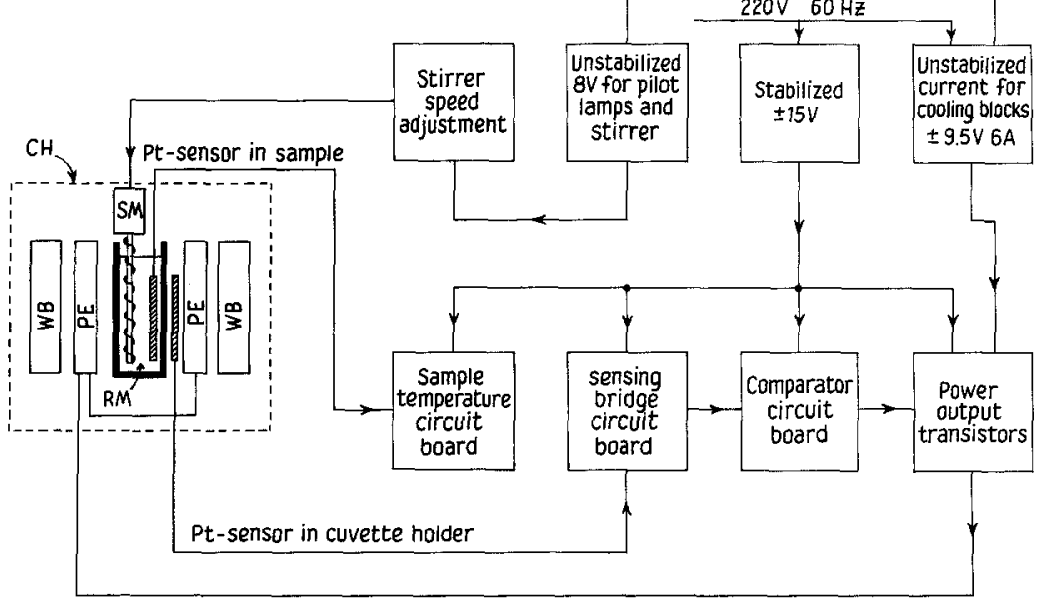

Fig. 1. Schematic diagram of the complete instrument. The cuvette holder assemply $(\mathrm{CH})$ (or the $\mathrm{O}_{2}$-electrode assembly, not shown) includes water-cooled Al blocks (WB), a Pt temperature sensor, and two Peltier elements (PE) in contact with the cuvette. The cuvette contains the reaction mixture, a stirrer connected to a motor (SM), and a Pt temperature sensor.

Arrhenius profiles. A major difficulty is the amount of time required to equilibrate a refrigerated water bath, the usual temperature controller, at up to 15 temperatures between 0 and $40^{\circ}$. Although direct electrical heating could rapidly achieve and control sample temperatures above ambient level, available methods for cooling are much slower.

In studies on the membrane dependence of cytochrome $b_{5}$ reoxidation rates in rat liver microsomes (Hoch et al., 1980) a Pelticr-effect temperature controller was developed for use in spectrophotometric measurements. Peltier in 1834 found that current passing through a bimetallic junction changes the temperature. This small effect is amplified by the use of a series of semiconductor materials to compose a block, one side of which cools and the other heats when current passes in one direction (Siemens AB). ${ }^{3}$ Current reversal produces thermal reversal. A steady current keeps cooling or heating power constant at a level that depends on current strength and polarity. Advantages pointed out for Peltier blocks (Siemens AB) include smallness and lightness, so that they can cool or heat rapidly where space is limited; freedom from maintenance (no moving parts); noiselessness; and insensitivity to shock,

\footnotetext{
${ }^{3}$ Similar units are available from the Cambridge Thermionic Corporation, Cambridge, Massachusetts 02138 .
} 
vibration, or position. When used in the present apparatus in conjunction with $\mathrm{Pt}$ temperature sensors, steady sample temperatures are achieved within 2-5 min and maintained within less than $\pm 0.04^{\circ} \mathrm{C}$. Temperature-controlled modules are described for a spectrophotometric cuvette and for an oxygen electrode, but the principle can be applied to a variety of specific designs.

\section{Materials}

The instrument consists of three parts (Fig. 1).

A. Cuvette Holder. The cuvette holder in Fig. 2 is designed as an accessory to an Aminco-Chance dual wavelength spectrophotometer. The surfaces of two Peltier blocks (Fig. 2H) are in firm thermal contact on the inside with a standard $1-\mathrm{cm}$ pathlength, 3-ml volume cuvette, and on the outside with an aluminum block that is cooled with tap water (about 0.5 liter/min) to transport excess heat (Fig. 2J, K). A recess in the block (Fig. 2D)

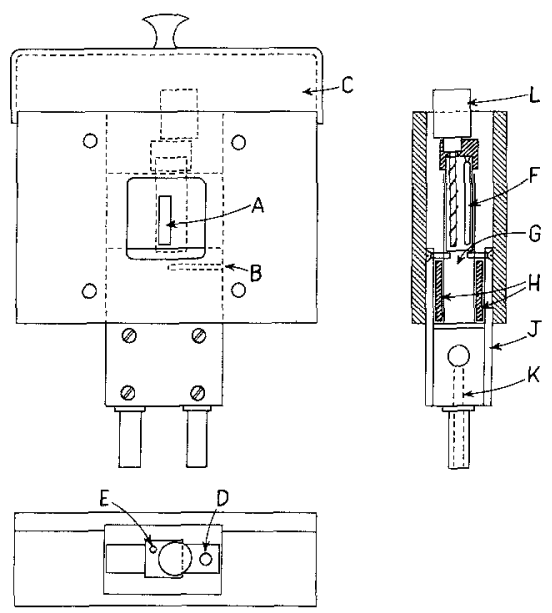

Fig. 2. Cuvette holder module. (A) Light beam aperture; (B) hole for Pt temperature sensor; (C) cover to exclude light; (D) hole in the Al block for calibrating thermometer; (E) access hole in the plastic cover that holds the stirrer and sensor in the cuvette; (F) Pt temperature sensor in the cuvette; $(G)$ cuvette holder in contact with $H$, the Peltier units, and $\mathbf{J}$, the Al block containing the Peltier elements, with cooling fingers for circulating cold water (K); (L) stirrer motor set in the plastic cap over the cuvette, with the stirrer blade in the reaction mixture; the stirrer blade and $\mathrm{F}$ are positioned to clear the light aperture $\mathrm{A}$. For scale reference, the inside dimensions of the cuvette are $1 \times 1 \mathrm{~cm}$. Not shown in the drawing are the electrical connections for the safety switches, the stirring motor, the two Pt temperature sensors, and the Peltier elements. The apertures for the light beam are blade springs that firmly position the cuvette and provide good thermal contact. 
fits the bulb of a calibrating Hg-glass thermometer. Two electric safety switches (not shown in the diagram) are in contact with the Peltier elements and break the current when the temperature exceeds $70^{\circ}$; they are necessary because the elements are damaged when allowed to stand at $90^{\circ} \mathrm{C}$ for $10 \mathrm{~min}$, which might occur if the cooling water is forgotten or interrupted. The current flow into the Peltier blocks is controlled by a $100-\Omega \mathrm{Pt}$ temperature sensor sealed in the cuvette holder (Fig. 2B).

B. Sample Temperature Sensor Unit. This unit is replaceable on top of the cuvette, and contains a $\mathrm{Pt}$ temperature sensor and a stirring rod driven by a miniature motor of adjustable speed (Fig. 2F, L). The screw-shaped stirrer prevents settling of particulate suspensions and speeds temperature exchange between the cuvette holder and the sample. A separate switch in the stirrer speed adjustment circuit (Fig. 1) can turn off the stirrer after the reaction is started, in cases where particle settling is not a problem. The sample temperature signal is amplified and read off a panel meter (Fig. 3, $\mathrm{C}^{\circ}$ ) that can be set to cover one of five $10^{\circ}$ scales between 0 and $50^{\circ}$ (Fig. 3). The sample temperature can also be read on a recorder set to $30 \mathrm{mV}$ full scale. Both the sensor-stirrer unit and the cuvette are removable for cleaning. However, to speed up readings the cuvette can be evacuated and filled or receive additions of solution with the unit in situ, through a hole in the unit top (Fig. 2E).

C. Electronic Control Unit. This unit (Fig. 4) comprises the following: (a) a sensing bridge with input from the $\mathrm{Pt}$ temperature sensor in the cuvette holder, a $\pm 15 \mathrm{~V}$ stabilized source, and a 10-turn helipot (Fig. 4, R4) for setting temperature; (b) a comparator circuit that controls a $\pm 9.5 \mathrm{~V}, 6 \mathrm{~A}$ unstabilized current that passes through two power-output transistors $\left(T_{1}, T_{2}\right.$ in Fig. 4) to supply heating and cooling power for the two Peltier blocks $\left(\mathrm{P}_{1}\right.$ and $\mathrm{P}_{2}$ ); a panel meter shown above $\mathrm{P}_{1}$ and $\mathrm{P}_{2}$ indicates whether heating or cooling is proceeding. Switch E1 is used for protection against excessively high temperatures and Switch E2, which is situated on the front panel, can turn off the Peltier units to allow the use of the instrument as a sensitive thermometer only.

D. $\mathrm{O}_{2}$-Electrode Holder. This unit (Fig. 5) can be used to obtain Arrhenius plots of respiration. Like the cuvette holder, it includes two Peltier units in contact with water-cooled $\mathrm{Al}$ blocks, and a Pt temperature sensor that feeds into the sensing bridge and comparator circuits. A separate stirring motor with a magnetic head mixes the sample through a magnetic flea. The sample, in a glass tube, fits into the Al block, with thermal contact improved through a layer of added glycerin. Another similar vessel in the Al block is used to store and temperature-equilibrate additional reaction mixture for the next run. Fitting into the reaction vessel are a Clark $\mathrm{O}_{2}$ electrode (Yellow 


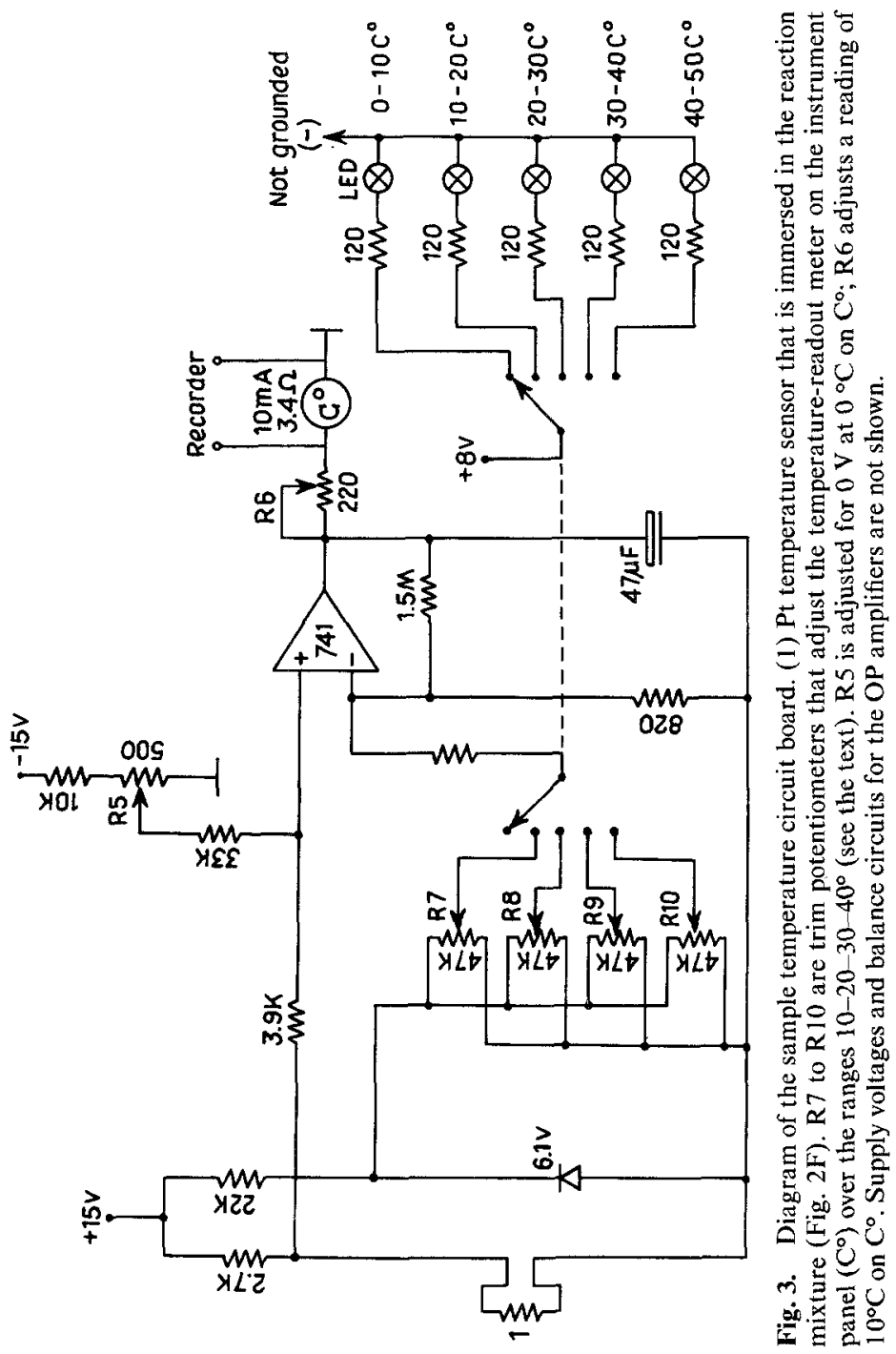




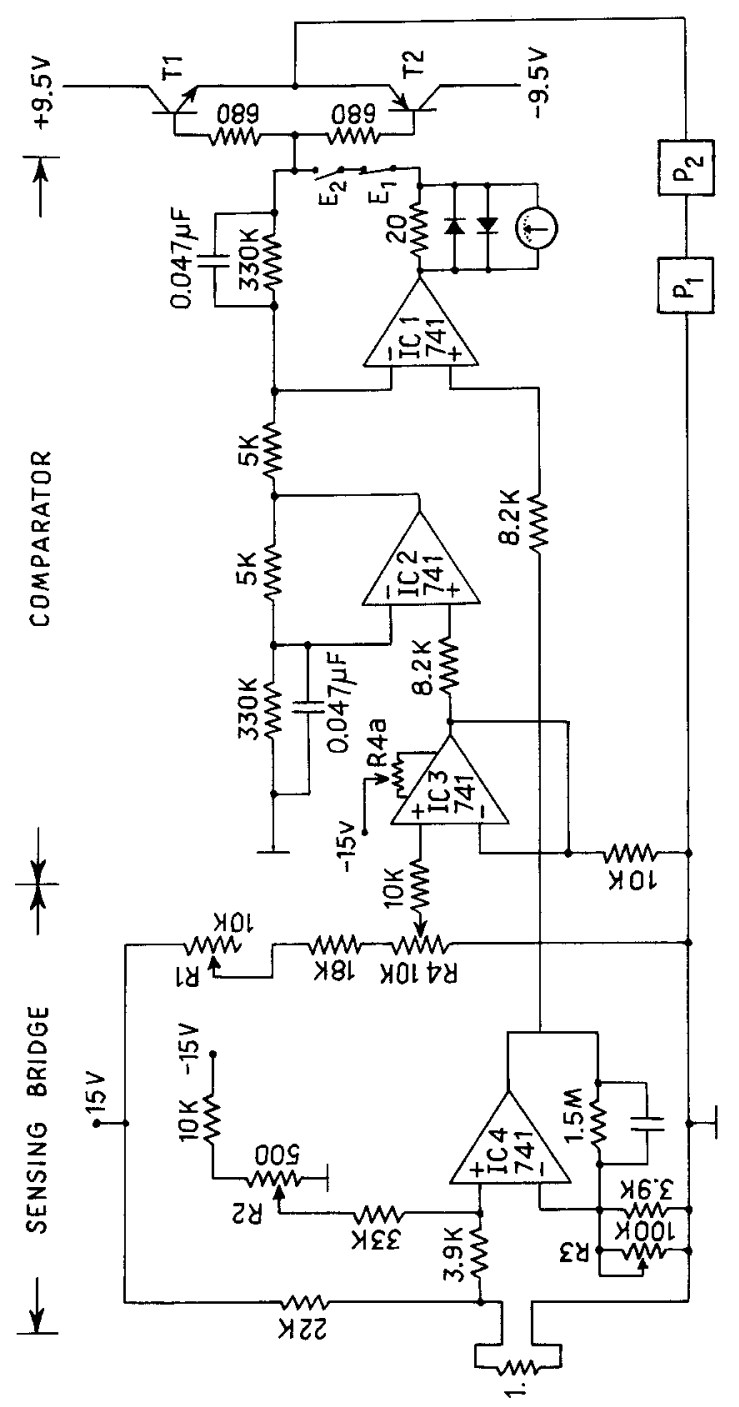

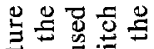

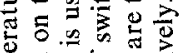
要

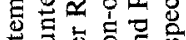

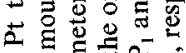
- 可

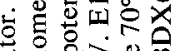
呟电

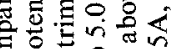
용요

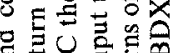

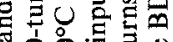
○吉主

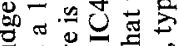

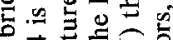
曲芯云兽 它总卷 总氖氖 을 을 क 넝 응

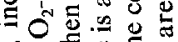
ธิษ 혼

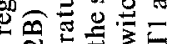
N 的至至焉 类

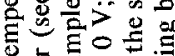
与 ᄂ 융용

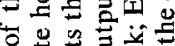
苍出

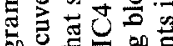
Do 0 잉

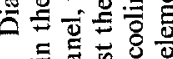
드로요요

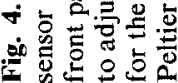




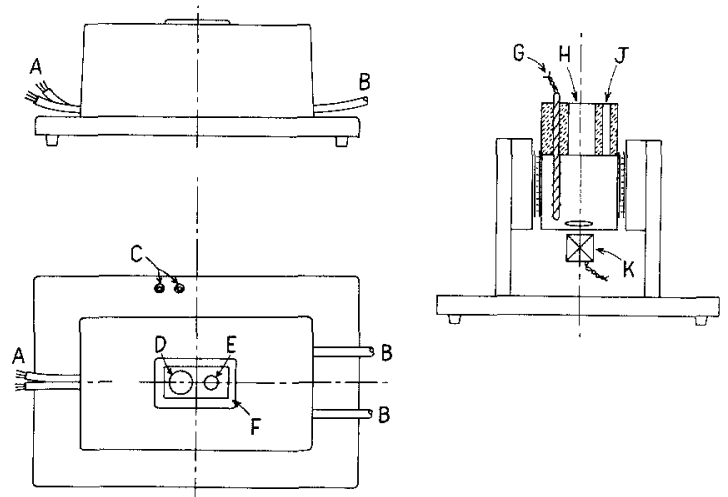

Fig. 5. $\mathrm{O}_{2}$-electrode holder module. (A) Electrical connections; (B) hoses for cooling water flow; (C) connectors for the $\mathrm{Pt}$ temperature sensor (G) that is immersed in the 2-ml reaction vessel that fits into the aperture (D) in the Al block; (E) vessel aperture in the block for pre-equilibrating the reaction mixture; $(F)$ rubber seal between the Al block and the unit casing; $(\mathrm{H})$ aperture in the plastic cap that fits into the reaction vessel; $(\mathrm{H})$ assembly that accommodates an $\mathrm{O}_{2}$ electrode (not shown), holds $\mathrm{G}$, and has an additional narrow aperture $(\mathrm{J})$ that serves to eliminate air bubbles from the reaction mixture and to permit access for filling and evacuation; (K) magnetic stirrer motor; the stirring flea in the reaction mixture is shown above. A calibrating $\mathrm{Hg}$-glass thermometer can be inserted through $\mathrm{H}$ in place of the $\mathrm{O}_{2}$ electrode. The Peltier units and the Al blocks are shown in contact with the reaction vessel but are not labeled. For scale reference, the diameter of the aperture $\mathrm{D}$ in the Al block is $2 \mathrm{~cm}$.

Springs, Ohio) and a Pt temperature sensor; their outputs, suitably amplified, are recorded as $\left[\mathrm{O}_{2}\right]$ and sample temperature respectively.

\section{Temperature Calibration}

The right side of the sensing bridge (Fig. 4) is first adjusted as follows. Connect a voltmeter on the output of IC3 pin 6. Turn the 10-turn potentiometer (R4 in Fig. 4, mounted on the front panel), which sets the sample temperature, fully counterclockwise. The voltage across the output of IC3 should be zero, if it is not $0 \mathrm{~V}$, adjust the balance of IC3 (with the trimmer potentiometer, $\mathrm{R} 4 \mathrm{a}$ ). When the 10-turn temperature-setting potentiometer $\mathrm{R} 4$ is turned fully clockwise, the voltage should be $+5.0 \mathrm{~V}$, which can be set by adjusting $\mathrm{R} 1$. The left side of the sensing bridge is adjusted against a calibrating glass-Hg thermometer which is placed in the drilled hole in the block (Fig. 2D) together with ethanol to provide thermal contact. When the temperature-setting potentiometer R4 is set fully counterclockwise, the block cools; down-adjust R2 (Fig. 4) until the thermometer equilibrates at $0.0^{\circ}$. Set 
$\mathrm{R} 4$ to $10^{\circ}$ equal to one turn, and adjust $\mathrm{R} 3$ until the block temperature is steady at $10.0^{\circ}$. The temperature setting should then be alternated several times between 0.0 and $10.0^{\circ}$, adjusting R 2 and R 3 successively to correct the voltage for the $10^{\circ}$ span.

Now the sample temperature bridge circuits (Fig. 3) can be calibrated to give correct readout either on the panel meter $\left(\mathrm{C}^{\circ}\right.$ in Fig. 3 ) or on a recorder. With the temperature set at $0.0^{\circ}$, and the temperature readout decade setting at $0-10^{\circ}$, adjust R 5 until the panel meter reads $0.0^{\circ}$. In succession, adjust R6 to give a reading of $10^{\circ}$ (decade setting at $10-20^{\circ}$ ) when the temperature of the block is set at $10^{\circ}$; set at $20^{\circ}$ and adjust $\mathrm{R} 7$ to give a correct readout; and so on through $\mathrm{R} 8, \mathrm{R} 9$, and $\mathrm{R} 10$ to $50.0^{\circ}$. The temperature readout settings are quite stable. The temperature calibration settings may show slight variations, and it is well to check the temperature readings with the calibrating glass- $\mathrm{Hg}$ thermometer each day.

\section{Operation}

The temperature of a stirred 3-ml reaction mixture in the cuvette holder was recorded to demonstrate how fast the sample temperature reaches a new steady level, how repeatable new temperature settings are, and how variable steady sample temperatures are (Fig. 6). In the left tracing in Fig. 6, the starting sample temperature is set to $11.1^{\circ}$. Increasing the temperature setting by 30 units (out of 1000 units on the 10-turn helipot) increases sample temperature to $12.3^{\circ}$ in 3 to $5 \mathrm{~min}$; to accelerate sample warming the
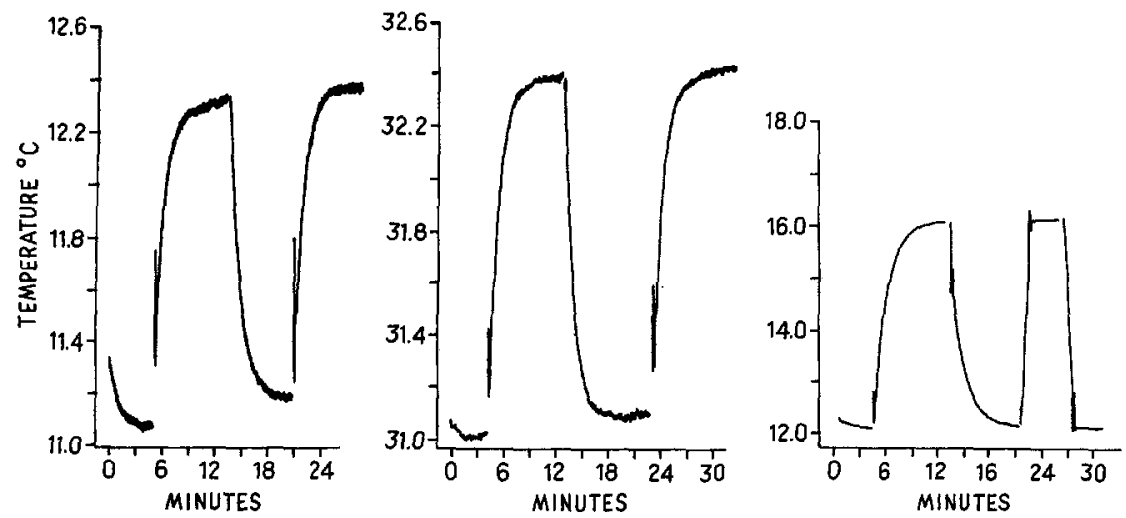

Fig. 6. Recordings of sample temperature changes in response to new settings on the temperature-controlling potentiometer (R4 in Fig. 4). The procedures are described in the text. 
setting was held at about 100 units higher than the original position for a few seconds, which produced the transient rapid rise in the sample temperature. The reproducibility of the sample temperature is $0.04^{\circ}$ or less. The center tracing in Fig. 6 shows similar changes between 31 and $32^{\circ}$, where temperature noise is slightly less than in the lower temperature range. The right tracing in Fig. 6 shows changes in sample temperatures when the settings are changed by 100 units. A $4^{\circ}$ change takes about 5 min when the setting is overshot briefly; in the second cycle the overshoot was maintained until the actual sample temperature was within $1^{\circ}$ of that desired. In this way, a steady sample temperature $4^{\circ}$ different from the original one is attained in about 1 $\min$.

In measuring enzyme activities, when the stock reaction mixture is kept on ice or at room temperature, a heating or cooling rate of about $4^{\circ} / \mathrm{min}$ can be expected in achieving a steady temperature before the reaction is started. To shorten this period, the block for the $\mathrm{O}_{2}$ electrode has an extra hole for a vessel that can contain reaction mixture (Fig. 5E) that pre-equilibrates for the next assay, which usually involves a temperature difference of only 2 or $3^{\circ}$.

Figure 7A shows a typical Arrhenius plot obtained with one batch of rat liver mitochondria, in which $\mathrm{Ca}^{2+}$ uptake rates were measured spectrophotometrically. In contrast to the inflection around $20^{\circ}$ in the Arrhenius profile that we and others see for membrane-dependent processes in mitochondria from normal rats (Raison, 1973; Fox, 1975), the mitochondria obtained from triiodothyronine-injected rats show a linear profile between 37 and $9^{\circ}$, suggesting altered membrane dependence. The triangle symbols show that mitochondrial aging has not affected this Arrhenius profile. Figure 7B is an Arrhenius plot of mitochondrial respiration rates, measured with the $\mathrm{O}_{2}$ electrode module. As indicated by the presence of a transition temperature, membrane dependence differs for the energy-linked (Stage 3) and energyindependent (State 4) modes of respiration in these heart mitochondria.

\section{Discussion}

It may not be immediately obvious why the units contain two Pt temperature sensors, one embedded in the reaction mixture holder that regulates temperature to a preset level, and the other immersed in the sample that measures temperature. The removal of the sample sensor, or the replacement of the reaction mixture with a fresh one for a rate determination at a new temperature, will produce a large change in signal from the sample sensor. These changes preclude its concomitant use as the temperature 

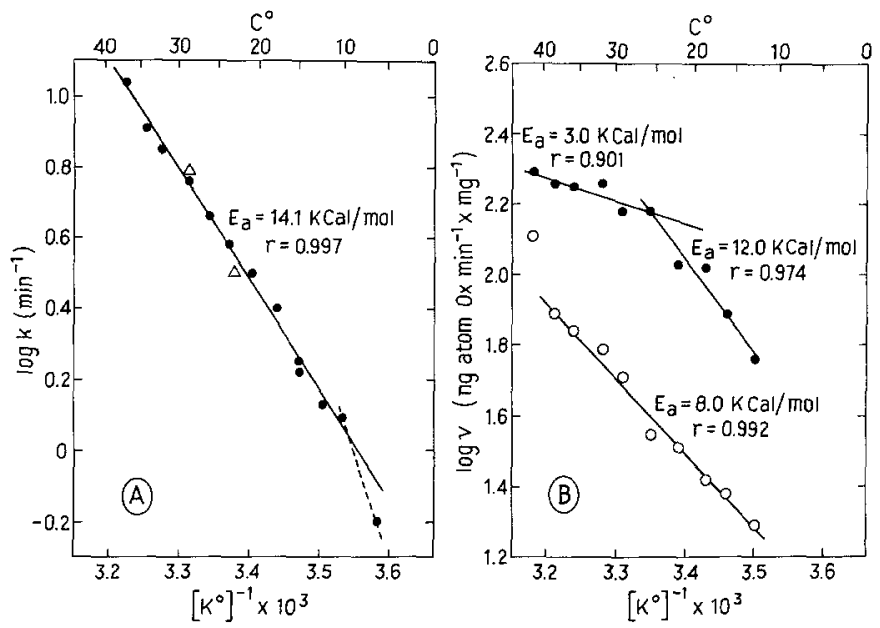

Fig. 7. (A) An Arrhenius plot of respiration-driven $\mathrm{Ca}^{2+}$ uptake by mitochondria obtained from the liver of a hyperthyroid rat (J. O. Peabody and F. L. Hoch, unpublished data). Uptake rates were measured spectrophotometrically through the change in absorbance at $540-507 \mathrm{~nm}$ (Mela and Chance, 1968), using an Aminco-Chance dual-wavelength spectrophotometer. First-order initial rate constants $k$, were calculated from the tracings. The solid circles are $\log k$ values measured consecutively at temperatures between 37 and $9{ }^{\circ} \mathrm{C}$, plotted against the reciprocal absolute temperature, $\left[K^{\circ}\right]^{-1}$; a least-mean-square line and correlation coefficient $r$ were calculated for all the points except that at $9.3^{\circ} \mathrm{C}$, which may represent a point on a line with a different slope, $E_{a}$. The triangle symbols are $\log k$ values measured 90 min after the start of the experiment; they represent the effects of aging upon the iced mitochondrial suspension. (B) Arrhenius plots of $\mathrm{O}_{2}$ consumption by mitochondria obtained from rat hearts. $\mathrm{O}_{2}$ concentrations were measured with an $\mathrm{O}_{2}$ electrode (Shaw and Hoch, 1977), with temperature controlled in the module shown in Fig. 5. Respiratory rates $v$ were calculated in the presence of ADP $+P_{i}$ (state 3, solid symbols) and after ATP synthesis stopped (State 4 , open symbols). The $\log v$ values are plotted against $\left[K^{\circ}\right]^{-1}$. Least-mean-square lines and $r$ values are shown for all the State 3 points, and for all the State 4 points except that at about $41^{\circ} \mathrm{C}$, which probably represents loose coupling of respiration. Total time for the measurement of these rates was $200 \mathrm{~min}$.

regulator, because they would effect unnecessarily large temperature changes in the Peltier blocks, and time would be wasted in correcting the overshoot. With the temperature regulator permanently placed in the block outside the reaction mixture, new temperature settings can be attained rapidly despite the variations in the temperature of the sample sensor. The time saved in this way can be shortened further by storing reaction mixture in the regulated block rather than at room temperature or in ice. Only the sample temperature is, of course, applicable for the Arrhenius plot, and with good thermal contact between the stirred sample and the Peltier block new temperatures can be adjusted to within $0.1^{\circ}$ at about $4^{\circ} / \mathrm{min}$, as shown in Fig. 6.

Peltier elements for heating and cooling in a confined space were used by 
Neupert et al. (1972) in a microscope stage. These authors mentioned potential applications to biological and medical studies involving phase transitions. A number of solid-state temperature regulators have since become commercially available, but they do not have the temperature readout and feedback control features described here.

\section{Acknowledgments}

F. L. Hoch is grateful to Professor L. Ernster for his advice and hospitality.

\section{References}

Fox, C. F. (1975). In MTP International Review of Science: Biochemistry, Series 1 (Fox, C. F., ed.), Butterworths, London, pp. 279-306.

Hoch, F. L., DePierre, J. W., and Ernster, L. (1980). Eur. J. Biochem., 109, 301-306.

Mela, L. T., and Chance, B. (1968). Biochemistry 11, 4059-4063.

Neupert, M., Seifarth, H. H., Herrling, R., and Hahne, B. (1972). Jena Rev. 17, 75-83.

Raison, J. K. (1973). J. Bioenerg. 4, 285-309.

Shaw, M. J., and Hoch, F. L. (1977). J. Mol. Cell. Cardiol. 9, 749-761.

Siemens AB, Avdelning Telekomponenter, Fack 10435 Stockholm 23, Sweden: Peltier Cooling Block PKE 36 A 001. 